\title{
Clinical Pharmacokinetics and Pharmacodynamics of Evolocumab, a PCSK9 Inhibitor
}

\author{
Sreeneeranj Kasichayanula ${ }^{1} \cdot$ Anita Grover $^{1} \cdot$ Maurice G. Emery $^{1}$. \\ Megan A. Gibbs ${ }^{1} \cdot$ Ransi Somaratne $^{2} \cdot$ Scott M. Wasserman $^{2} \cdot$ John P. Gibbs $^{1}$
}

Published online: 20 January 2018

(C) The Author(s) 2018. This article is an open access publication

\begin{abstract}
Proprotein convertase subtilisin/kexin type 9 (PCSK9) increases plasma low-density lipoprotein cholesterol (LDL-C) by decreasing expression of the LDL receptor on hepatic cells. Evolocumab is a human monoclonal immunoglobulin G2 that binds specifically to human PCSK9 to reduce LDL-C. Evolocumab exhibits nonlinear kinetics as a result of binding to PCSK9. Elimination is predominantly through saturable binding to PCSK9 at lower concentrations and a nonsaturable proteolytic pathway at higher concentrations. The effective half-life of evolocumab is 11-17 days. The pharmacodynamic effects of evolocumab on PCSK9 are rapid, with maximum suppression within $4 \mathrm{~h}$. At steady state, peak reduction of LDL-C occurs approximately 1 week after a subcutaneous dose of $140 \mathrm{mg}$ every 2 weeks (Q2W) and 2 weeks after a subcutaneous dose $420 \mathrm{mg}$ once monthly (QM), and returns towards baseline over the dosing interval. In several clinical studies, these doses of evolocumab reduced LDL-C by approximately 55-75\% compared with placebo. Evolocumab also reduced lipoprotein(a) [Lp(a)] levels and improved those of other lipids in clinical studies. No clinically meaningful differences in pharmacodynamic
\end{abstract}

Former Amgen employee: Sreeneeranj Kasichayanula, Anita Grover, Maurice G. Emery, Megan A. Gibbs, John P. Gibbs.

Electronic supplementary material The online version of this article (https://doi.org/10.1007/s40262-017-0620-7) contains supplementary material, which is available to authorized users.

Sreeneeranj Kasichayanula

neeranj@gmail.com

1 Clinical Pharmacology Modeling and Simulation, Amgen Inc., Thousand Oaks, CA, USA

2 Clinical Development, Amgen Inc., Thousand Oaks, CA, USA effects on LDL-C were observed in adult subjects regardless of mild/moderate hepatic impairment, renal impairment or renal failure, body weight, race, sex, or age. No clinically meaningful differences were observed for the pharmacodynamic effects of evolocumab on LDL-C between patients who received evolocumab alone or in combination with a statin, resulting in additional lowering of LDL-C when evolocumab was combined with a statin. No dose adjustment is necessary based on patient-specific factors or concomitant medication use.

\section{Key Points}

Evolocumab, a human monoclonal immunoglobulin that binds specifically to human proprotein convertase subtilisin/kexin type 9 (PCSK9) on hepatic cells to reduce low-density lipoprotein cholesterol (LDL-C), exhibits nonlinear kinetics and a half-life of 11-17 days.

Maximal suppression of PCSK9 occurs within $4 \mathrm{~h}$, and peak reduction of LDL-C, ranging from 55 to $75 \%$, occurs approximately $1-2$ weeks after a dose of evolocumab.

Patient-specific factors do not have a clinically meaningful effect on the pharmacodynamic effects of evolocumab; thus, no dose adjustment is necessary based on patient-specific factors or concomitant medication use. 


\section{Introduction}

Cardiovascular disease is the leading cause of death and disability globally $[1,2]$. Dyslipidemia is a major, common, and modifiable risk factor for cardiovascular disease $[1,2]$. Randomized clinical studies aimed at lowering lowdensity lipoprotein cholesterol (LDL-C) show a consistent relationship between LDL-C reduction and cardiovascular risk reduction [3], particularly for treatments that act through the LDL-C receptor (LDLR) [4]. The relationship between lower LDL-C and reduction of major cardiovascular events extends to the lowest limit of LDL-C tested, with no evidence of attenuation of cardiovascular benefits at very low levels of LDL-C [4].

Statins, the first-line treatment for primary hyperlipidemia and mixed dyslipidemia, reduce both LDL-C (30-40\% with standard doses [5]) and cardiovascular events [3, 4]. However, in clinical practice, approximately one in four patients with hyperlipidemia, including one in six low-risk patients and one in three high-risk patients, continues to have elevated LDL-C and does not achieve traditional treatment goals with statin therapy [6]. Less than $50 \%$ of patients are adherent to statin therapy at 1 year [7]. Some of the nonadherence in clinical practice is attributable to statin intolerance, most commonly because of myopathy, which can lead to either lower doses or complete discontinuation of statin therapy [8].

Few established medical treatment options significantly reduce cardiovascular events among patients who require additional LDL-C lowering after an adequate trial of statin therapy. Among patients with a history of acute coronary syndromes, adding a second lipid-lowering therapy (ezetimibe) to a statin lowered mean LDL-C to $53.7 \mathrm{mg} / \mathrm{dl}$ compared with $69.5 \mathrm{mg} / \mathrm{dl}$ with a statin alone (a relative LDL-C reduction of $23 \%$ ), and the combination significantly reduced the incidence of cardiovascular outcomes compared with a statin alone [9].

An enhanced understanding of the regulation of LDL-C led to the development of targeted therapy to lower LDL-C via novel mechanisms. Recycling of the LDLR to the surface of normal hepatic cells regulates plasma LDL-C $[10,11]$. Proprotein convertase subtilisin/kexin type 9 (PCSK9) is a serine protease that decreases expression of LDLRs on hepatic cells, leading to increased plasma LDLC (Fig. 1) [12, 13]. Human genetic studies identified gainof-function mutations in the PCSK9 gene that are associated with elevated serum LDL-C levels and premature coronary heart disease and also identified other loss-offunction mutations in the PCSK9 gene that are associated with low serum LDL-C levels [14-17].

Evolocumab (REPATHA ${ }^{\circledR}$, Amgen, Inc.) is a human monoclonal immunoglobulin G2 (IgG2) that binds specifically to human PCSK9 to inhibit its effects on LDLR, resulting in reduction of LDL-C. In randomized placebo-controlled clinical studies, evolocumab doses of $140 \mathrm{mg}$ every 2 weeks (Q2W) or $420 \mathrm{mg}$ once monthly (QM) for 12 weeks reduced LDL-C by approximately $55-75 \%$, compared with placebo, at the mean of weeks 10 and 12 among patients with primary hyperlipidemia and mixed dyslipidemia who received evolocumab either as monotherapy $[18,19]$ or in combination with a statin $[20,21]$ and in patients with heterozygous familial hypercholesterolemia $(\mathrm{HeFH})$ who received evolocumab with a statin $[22,23]$. In randomized active-control studies, these doses of evolocumab for 12-24 weeks reduced LDL-C by approximately $35-45 \%$ compared with ezetimibe in patients with primary hyperlipidemia and mixed dyslipidemia $[18,19]$, including patients with documented statin intolerance [24-26]. Robust reductions in LDL-C persisted through 1 year of evolocumab therapy in a placebo-controlled study [27] and two active-controlled studies versus standard of care [28]. Evolocumab also reduced LDL-C compared with placebo when added to other lipid-lowering therapy in patients with homozygous familial hypercholesterolemia $(\mathrm{HoFH})$ [29, 30]. The safety profile for evolocumab was generally similar to that for control (placebo or ezetimibe) in each of these studies, and in a pooled analysis of efficacy and safety across four studies [31]. A randomized, double-blinded study of patients with coronary disease who were treated with evolocumab $420 \mathrm{mg}$ QM or placebo for 18 months showed that a $60 \%$ mean reduction in LDL-C in the evolocumab group was associated with regression of coronary atherosclerosis on intravascular ultrasound [32].

Evolocumab was approved in the EU in July 2015 for use in the following populations: in combination with a statin, with or without other lipid-lowering therapies, in adults with primary hypercholesterolemia $(\mathrm{HeFH}$ or nonfamilial hypercholesterolemia) or mixed dyslipidemia who are unable to reach LDL-C goals with the maximum tolerated dose of a statin; or alone or in combination with other lipid-lowering therapies in patients who are statin intolerant or for whom a statin is contraindicated. Evolocumab was also approved for use in the EU in adults and adolescents aged $\geq 12$ years with $\mathrm{HoFH}$ in combination with other lipid-lowering therapies. In the USA, evolocumab was approved in August 2015 for use in combination with maximally tolerated statin therapy for adults with $\mathrm{HeFH}$ or clinical atherosclerotic cardiovascular disease who require additional lowering of LDL-C, or in combination with other LDL-lowering therapies (e.g., statins, ezetimibe, LDL apheresis) in patients with HoFH who require additional lowering of LDL-C. This review summarizes the clinical pharmacokinetic and pharmacodynamic properties of evolocumab. 

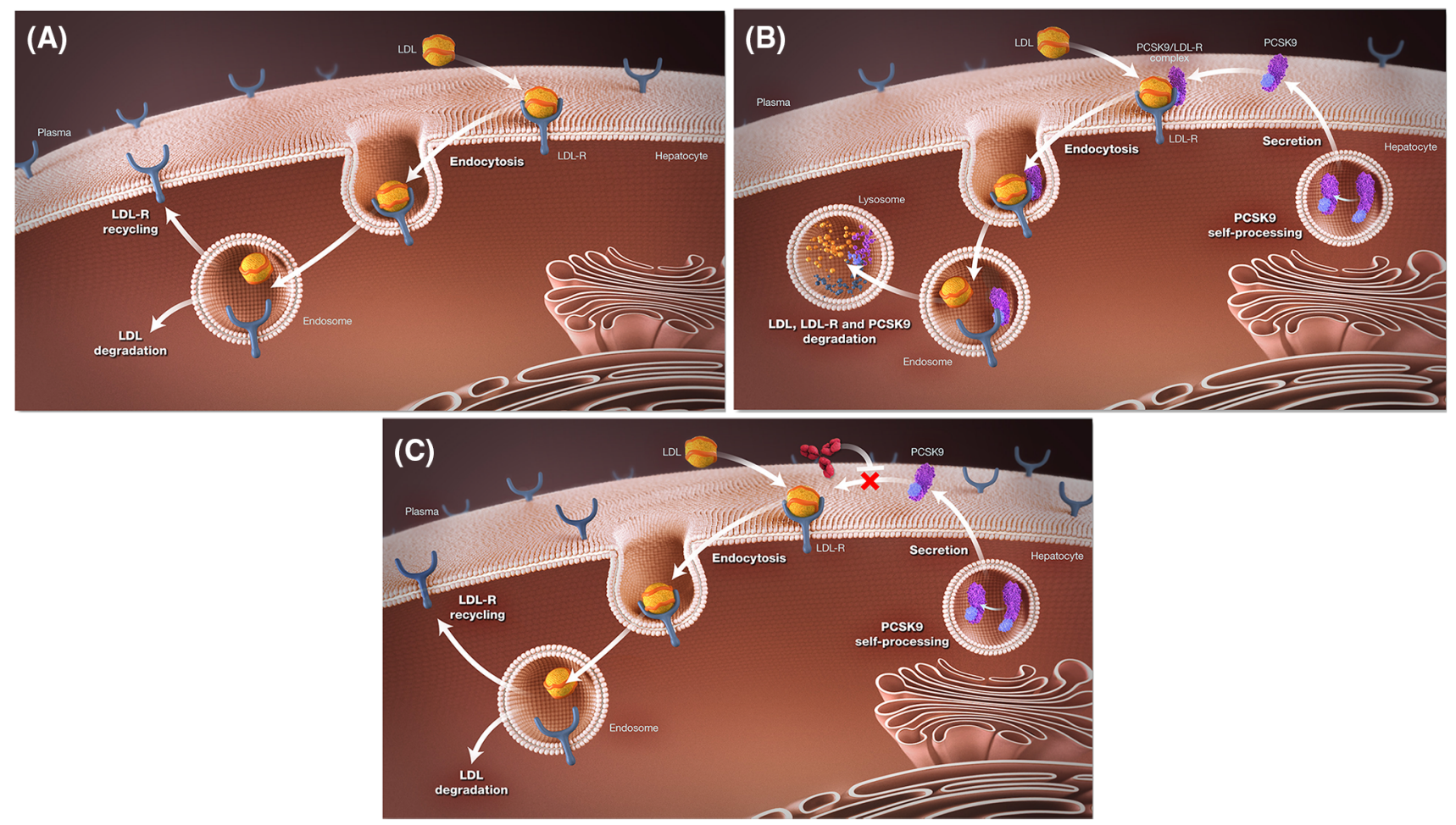

Fig. 1 Mechanism of action: PCSK9 inhibition with evolocumab increases LDL-R and decreases serum concentrations of LDL-C $[10,12,13,33,52-55]$. a The liver is responsible for catabolism of plasma LDL. Hepatocytes express LDL-R that bind LDL and remove it from the plasma. Upon internalization, vesicles containing the LDL-LDL-R complex fuse with endosomes. LDL-R cycles back to the hepatocyte surface to bind additional LDL. Free LDL in endosomes is degraded into lipids, free fatty acids, and amino acids. b PCSK9 is a protein that regulates the expression of LDL-R in the liver. Hepatocytes produce a precursor of PCSK9 that undergoes selfcleavage in the endoplasmic reticulum and ultimately is secreted into plasma as functional PCSK9. Extracellular PCSK9 binds to LDL-Rs on the surface of the hepatocyte and is internalized with the LDL-

\section{Clinical Pharmacology}

\subsection{Structure and Physicochemical Properties}

Evolocumab is a human monoclonal $\mathrm{IgG} 2$ directed against human PCSK9 [33]. Binding of evolocumab to PCSK9 inhibits circulating PCSK9 from binding to the LDLR. This prevents PCSK9-mediated LDLR degradation and allows LDLR to recycle back to the hepatic cell surface, thereby lowering serum LDL-C concentrations (Fig. 1).

\subsection{Nonclinical Pharmacology}

Nonclinical studies were conducted to assess the pharmacokinetics and pharmacodynamics of evolocumab [33]. In vitro, evolocumab bound PCSK9 with high affinity, with equilibrium dissociation constants of 4,4 , and $160 \mathrm{pM}$, for human, cynomolgus, and mouse PCSK9, respectively.
LDL-R complex. The LDL-R-PCSK9 complex is routed to the lysosome for degradation, thereby preventing the cycling of LDL-R back to the hepatocyte surface. The reduced concentration of LDL-R on the surface of hepatocytes results in a lower rate of plasma LDL elimination. c A monoclonal antibody directed against PCSK9 could lower LDL if binding to circulating PCSK9 blocks the interaction of PCSK9 with cell surface LDL-R. Internalized LDL-R could cycle back to the cell surface instead of being degraded in lysosomes, leading to increased concentrations of LDL-R on the cell surface. This could result in a higher LDL elimination rate by hepatocytes and an overall reduction in plasma LDL. $L D L-C$ low-density lipoprotein cholesterol, $L D L-R$ low-density lipoprotein receptor, PCSK9 proprotein convertase subtilisin/kexin type 9

Evolocumab sterically hindered binding of PCSK9 to LDLR, with a half-maximal inhibition $\left(\mathrm{IC}_{50}\right)$ of $2.08 \pm 1.21 \mathrm{nM}$. Incubation of the statin mevinolin with HepG2 cells increased LDLR protein levels 1.6- to 2.3-fold compared with untreated cells; when evolocumab was added to mevinolin, LDLR protein levels increased 5.3- to 9.2-fold compared with untreated cells.

In mice, a single intravenous injection of evolocumab $10 \mathrm{mg} / \mathrm{kg}$ reduced total cholesterol by 20,26 , and $28 \%$ at 24, 72, and $144 \mathrm{~h}$ after injection, respectively [33]. The magnitude and duration of the effects of evolocumab were dose related: total cholesterol at 3 days after an injection of evolocumab 3,6 , or $10 \mathrm{mg} / \mathrm{kg}$ was reduced from baseline by 26,28 , and $36 \%$, respectively. By day 12 , total cholesterol levels in all treatment groups were similar to those in control animals. Administration of evolocumab in mice without LDLR did not significantly alter LDL-C 
concentrations, indicating that presence of the LDLR is required for lipid lowering with evolocumab therapy.

In cynomolgus monkeys with an average predose serum LDL-C of $72 \mathrm{mg} / \mathrm{dl}$, a single intravenous dose of evolocumab at $3 \mathrm{mg} / \mathrm{kg}$ lowered total cholesterol by a maximum of $48 \%$ at day 10 compared with an increase of $5 \%$ in control animals who received a single dose of a control antibody against keyhole limpet hemocyanin [33]. A larger treatment effect was seen for LDL-C, which decreased by $80 \%$ at day 10 in the evolocumab group and increased by $17 \%$ in the control group. Unbound PCSK9 decreased by $>97 \%$ by $15 \mathrm{~min}$ after the evolocumab injection, remained at this level through 3 days, and then gradually returned to baseline by 14 days.

\section{Clinical Pharmacokinetics}

\subsection{Data Sources}

A total of 26 global clinical studies provided information on the pharmacokinetic and pharmacodynamic properties of evolocumab in vivo. Eight were clinical pharmacokinetic studies with frequent sampling after a single dose or multiple doses of evolocumab by intravenous or subcutaneous administration in healthy subjects or patients with primary hyperlipidemia and mixed dyslipidemia [34, 35], in patients with hepatic impairment [36], or in patients with renal impairment [37]. Two were clinical biopharmaceutics studies of evolocumab by subcutaneous administration to demonstrate the pharmacokinetic equivalence of different product presentations. Supportive pharmacokinetics results were obtained from 16 phase II or phase III efficacy/safety studies with infrequent sampling in which evolocumab was administered subcutaneously Q2W or QM [18-30, 38]. The phase II studies generally collected samples for pharmacokinetics and pharmacodynamics at weeks $0,2,4,6,8$, 10 , and 12; a subset of patients in two of these studies $[18,20]$ participated in a pharmacokinetics/pharmacodynamics substudy and provided additional samples at weeks 9 and 11 . The phase III studies generally collected samples for pharmacokinetics and pharmacodynamics only at weeks $0,2,10$, and 12 .

\subsection{Sampling and Assays}

Venous blood samples for pharmacokinetic measurements were collected into tubes containing no anticoagulant and allowed to clot at room temperature for 30-60 min. Samples were then centrifuged, and serum aliquots were prepared and stored at $-80{ }^{\circ} \mathrm{C}$. Unbound evolocumab was measured in serum using a validated enzyme-linked immunosorbent assay with highly specific anti-idiotype antibodies for capture and detection of evolocumab. The validated procedure required accuracy and precision of \pm 15 and $\leq 15 \%$, respectively, for the standard, and \pm 20 and $\leq 15 \%$, respectively, for the quality control samples.

\subsection{Pharmacokinetic Overview}

Evolocumab exhibits nonlinear kinetics as a result of binding to PCSK9, predominantly evident at doses $<140 \mathrm{mg}$ [39, 40]. Subcutaneous administration of evolocumab $140 \mathrm{mg}$ in healthy volunteers resulted in a mean \pm standard deviation (SD) maximum drug serum concentration $\left(C_{\max }\right)$ of $18.6 \pm 7.3 \mu \mathrm{g} / \mathrm{ml}$ and mean $\pm \mathrm{SD}$ area under the concentration-time curve $\left(\mathrm{AUC}_{\text {last }}\right)$ of $188 \pm 98.6$ day $\cdot \mu \mathrm{g} / \mathrm{ml}$. Subcutaneous administration of evolocumab $420 \mathrm{mg}$ in healthy volunteers resulted in a mean $\pm \mathrm{SD} C_{\max }$ of $59.0 \pm 17.2 \mu \mathrm{g} / \mathrm{ml}$ and mean $\pm \mathrm{SD} \mathrm{AUC}_{\text {last }}$ of $924 \pm 346$ day $\cdot \mu \mathrm{g} / \mathrm{ml}$. Following a single intravenous dose of $420 \mathrm{mg}$, the mean \pm SD systemic clearance was estimated to be $12 \pm 2 \mathrm{ml} / \mathrm{h}$. An approximate two- to threefold accumulation was observed in mean \pm SD trough serum concentrations following subcutaneous administration of evolocumab $140 \mathrm{mg}$ Q2W ( $\left.C_{\min } 7.21 \pm 6.6\right)$ or $420 \mathrm{mg}$ QM $\left(C_{\min } 11.2 \pm 10.8\right)$, and trough serum concentrations approached steady state by 12 weeks of dosing.

\subsection{Absorption}

Following a single subcutaneous dose of evolocumab $140 \mathrm{mg}$ or $420 \mathrm{mg}$ administered to healthy adults, median peak serum concentrations were attained in 3-4 days, and estimated absolute bioavailability was $72 \%$. Dose proportional increases in exposure were observed with dose regimens of $\geq 140 \mathrm{mg}$.

\subsection{Distribution}

Following a single intravenous dose of evolocumab $420 \mathrm{mg}$, the mean $\pm \mathrm{SD}$ steady-state volume of distribution was estimated to be $3.3 \pm 0.51$, suggesting evolocumab has limited tissue distribution.

\subsection{Metabolism and Elimination}

Following a single intravenous dose of evolocumab $420 \mathrm{mg}$, the mean \pm SD systemic clearance was estimated to be $12 \pm 2 \mathrm{ml} / \mathrm{h}$. Evolocumab appeared to have two elimination phases [40]. At low concentrations, the elimination was predominately through saturable binding to the target (PCSK9). At higher concentrations, elimination was largely through a nonsaturable proteolytic pathway. Evolocumab was estimated to have an effective half-life of 11-17 days. 


\subsection{Immunogenicity}

Across 26 placebo-controlled and active-controlled clinical trials, $0.1 \%$ of patients treated with at least one dose of evolocumab tested positive for binding antibody development. None of these patients tested positive for neutralizing antibodies. There was no evidence that anti-drug binding antibodies affected the pharmacokinetic profile, clinical response, or safety of evolocumab. No dose adjustment is necessary based on immunogenicity. The long-term consequences of continuing evolocumab treatment in the presence of anti-drug binding antibodies are unknown.

\section{Pharmacodynamics}

\subsection{Inhibition of PCSK9}

A quantitative enzyme-linked immunosorbent assay (ELISA) was developed to evaluate the effects of evolocumab on unbound PCSK9 in human serum [41]. The precision and accuracy of the assay to detect endogenous PCSK9 was demonstrated, with coefficients of variation of $\leq 15 \%$ and bias of $\leq 20 \%$. Following single subcutaneous administration of evolocumab $140 \mathrm{mg}$ or $420 \mathrm{mg}$, maximum suppression of circulating unbound PCSK9 occurred by $4 \mathrm{~h}$ (Fig. 2). Unbound PCSK9 concentrations returned toward baseline when evolocumab concentrations decreased below the limit of quantitation. No increase in PCSK9 above baseline was observed during the washout of evolocumab (Fig. 2).

\subsection{Effects on Low-Density Lipoprotein Cholesterol (LDL-C) and Other Lipids}

A single subcutaneous administration of evolocumab $140 \mathrm{mg}$ or $420 \mathrm{mg}$ resulted in a reduction in LDL-C, with peak LDL-C reduction by 14 and 21 days, respectively (Fig. 2). No increase in LDL-C above baseline was observed during the washout of evolocumab (Fig. 2). With repeated doses, LDL-C levels declined and returned towards baseline over the dosing interval. The magnitude was dependent on both the dose and the dose interval; the nadir for LDL-C occurred at approximately 1 week after Q2W dosing and approximately 2 weeks after QM dosing [42].

In phase II and phase III clinical studies of patients with primary hyperlipidemia (familial or nonfamilial) and mixed dyslipidemia, evolocumab significantly reduced LDL-C [18-30, 38]. Evolocumab also increased highdensity lipoprotein cholesterol (HDL-C) and apolipoprotein A1 (ApoA1), and decreased total cholesterol and other lipids, in patients with primary hyperlipidemia and mixed
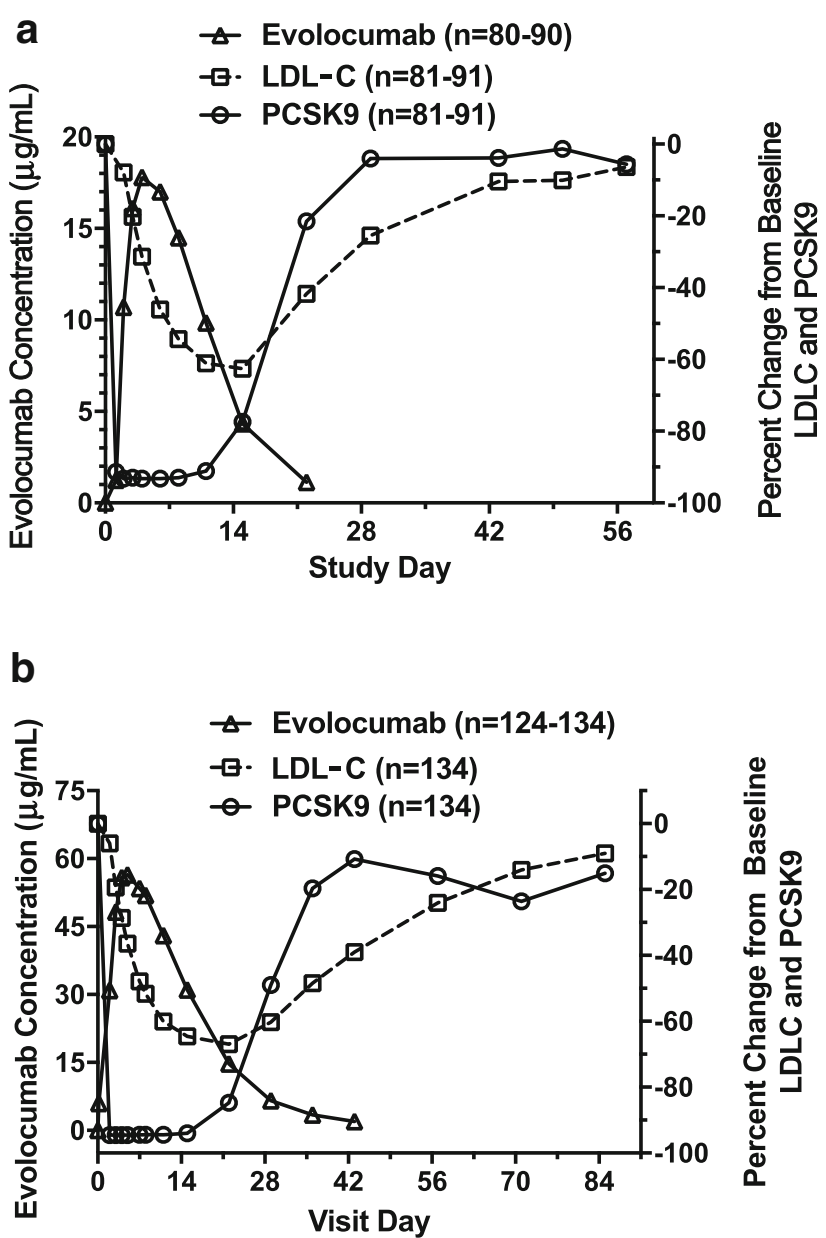

Fig. 2 Mean unbound evolocumab serum concentrations and geometric mean percent change from baseline in ultracentrifugation LDL-C and unbound PCSK9 in healthy subjects. a Single-dose SC evolocumab $140 \mathrm{mg}$. b Single-dose SC evolocumab $420 \mathrm{mg}$. $L D L-C$ low-density lipoprotein cholesterol, PCSK9 proprotein convertase subtilisin/kexin type 9, $S C$ subcutaneous. Amgen data on file

dyslipidemia [18-21, 24-28, 38] or HeFH [22, 23]. Across those studies, evolocumab reduced triglycerides less effectively than it reduced other lipids, but evolocumab has similar effects on LDL-C in patients with or without mixed dyslipidemia due to elevated triglycerides [43]. A pooled analysis of data across ten clinical studies determined that evolocumab reduced $\mathrm{Lp}$ (a) by approximately $20-25 \%$ in the randomized studies, and the reductions were maintained through week 52 of the open-label extension studies [44]. The reduction in $L p(a)$ was mediated in part by LDLR-mediated uptake [45]. The mechanism of action of PCSK9 monoclonal antibodies on lipoprotein metabolism was investigated in healthy volunteers using stable isotope tracer kinetics [46]. Evolocumab decreased the concentration of atherogenic lipoproteins, particularly LDL-C, by accelerating their catabolism. 


\subsection{Dose Selection}

The pharmacokinetic/pharmacodynamic relationship for evolocumab was described using a mathematical model that captured evolocumab binding and removal of unbound PCSK9 as well as reduction in LDL-C [40]. Data for this analysis were pooled from the first two phase I studies, including a single-dose study in healthy subjects and a multiple-dose escalation study in statin-treated patients with hypercholesterolemia. A target-mediated drug disposition model described the time course of unbound evolocumab concentrations and removal of unbound PCSK9. The estimated linear clearance and volume of distribution for evolocumab were $0.2561 /$ day and 2.661 , respectively. In this model, PCSK9 inhibited the elimination of LDL-C in a concentration-dependent manner. The concentration of unbound PCSK9 associated with IC $_{50}$ of LDL-C elimination was $1.46 \mathrm{nM}$. Thus, after administration of evolocumab, reduced levels of circulating PCSK9 led to enhanced elimination of LDL-C and an overall reduction in circulating LDL-C in serum. Based on simulations, $140 \mathrm{mg}$ Q2W and $420 \mathrm{mg}$ QM were predicted to achieve similar reductions in LDL-C, suggesting that an approximate threefold dose increase is required for a twofold extension in the dosing interval.

In an analysis of pooled data from two phase II studies, each of which included a pharmacokinetic and pharmacodynamic substudy with weekly blood samples at steady state (weeks 8-12), higher doses of evolocumab (140 mg or $420 \mathrm{mg}$ ) produced a greater and more sustained LDL-C response over the dosing interval than did a lower dose $(70 \mathrm{mg}$ ) [42]. The higher doses of evolocumab also produced a greater and more sustained reduction in unbound PCSK9 than the lower dose, consistent with the time course of LDL-C. Because a single measurement of lipids does not represent lipid-lowering accurately for an inhibitory monoclonal antibody administered Q2W or QM, the pooled analysis included lipid-lowering results from the end of study (week 12), the average of weeks 10 and 12, and the time-averaged effect (TAE) from weeks 8, 9, 10, 11, and 12. In these analyses, evolocumab $140 \mathrm{mg}$ Q2W and $420 \mathrm{mg}$ QM were therapeutically equivalent in LDL-C lowering and improvement in other lipids (Fig. 3), as well as safety and tolerability. Higher doses also reduced the variability in LDL-C response over the dosing interval than did lower doses. Thus, subcutaneous evolocumab $140 \mathrm{mg}$ Q2W and $420 \mathrm{mg}$ QM were chosen for the pivotal phase III studies.

\subsection{Effect of Baseline PCSK9 on LDL-C Response}

The effect of baseline PCSK9 on reductions in LDL-C were evaluated using pooled data from four phase III randomized clinical studies [47]. Across all quartiles of baseline PCSK9 concentrations, evolocumab $140 \mathrm{mg}$ Q2W and $420 \mathrm{mg}$ QM suppressed circulating PCSK9 levels by 90-100\% within 1 week of administration. Regardless of baseline PCSK9, both evolocumab $140 \mathrm{mg}$ Q2W and $420 \mathrm{mg}$ QM were associated with significant and consistent reductions in LDL-C.

\subsection{Effect on Cardiovascular Disease}

In a randomized placebo-controlled study of 27,564 patients with atherosclerotic cardiovascular disease and LDL-C $\geq 70 \mathrm{mg} / \mathrm{dl}$ who were receiving statin therapy, adding evolocumab $140 \mathrm{mg}$ Q2W or $420 \mathrm{mg}$ QM for a median of 2.2 years lowered LDL-C and significantly reduced the risk of the primary endpoint, which was a composite of cardiovascular death, myocardial infarction, stroke, hospitalization for unstable angina, or coronary revascularization [48]. A separate randomized placebocontrolled study of 968 patients with angiographic coronary disease showed that the addition of evolocumab resulted in a greater decrease in percent atheroma volume compared with placebo after 76 weeks of treatment [32].

\section{Pharmacokinetics and Pharmacodynamics in Specific Populations}

\subsection{Hyperlipidemic Patients vs Healthy Subjects}

In a cross-study comparison of pharmacokinetics among healthy subjects after a single dose of evolocumab and patients with hyperlipidemia after two doses of evolocumab, the $C_{\max }$ of unbound evolocumab was comparable between the two populations (Table 1). After adjustment for the number of doses, the AUC for unbound evolocumab was also comparable between healthy subjects and patients with hyperlipidemia (Table 1).

\subsection{Familial vs Non-Familial Hyperlipidemia}

The $C_{\max }$ and AUC for unbound evolocumab were not determined for patients with $\mathrm{HeFH}$ because the phase II study in this population [22] did not include a pharmacokinetics substudy. In phase III studies, similar serum evolocumab concentrations and pharmacodynamic effects were observed for patients with $\mathrm{HeFH}$ compared with the overall population of patients with primary hyperlipidemia and mixed dyslipidemia who were treated with a statin and evolocumab.

Patients with HoFH who did not require lipid apheresis could participate in a 12-week phase II/III study in which they received either evolocumab $420 \mathrm{mg}$ or placebo QM 
Fig. 3 Percent change (mean and $95 \%$ confidence interval) from baseline for lipid parameters: time-averaged effect, mean of weeks 10 and 12, and week 12. ApoAl apolipoprotein A1, ApoB apolipoprotein $\mathrm{B}, H D L-C$ highdensity lipoprotein cholesterol, $L D L-C$ low-density lipoprotein cholesterol, $Q 2 W$ once every 2 weeks

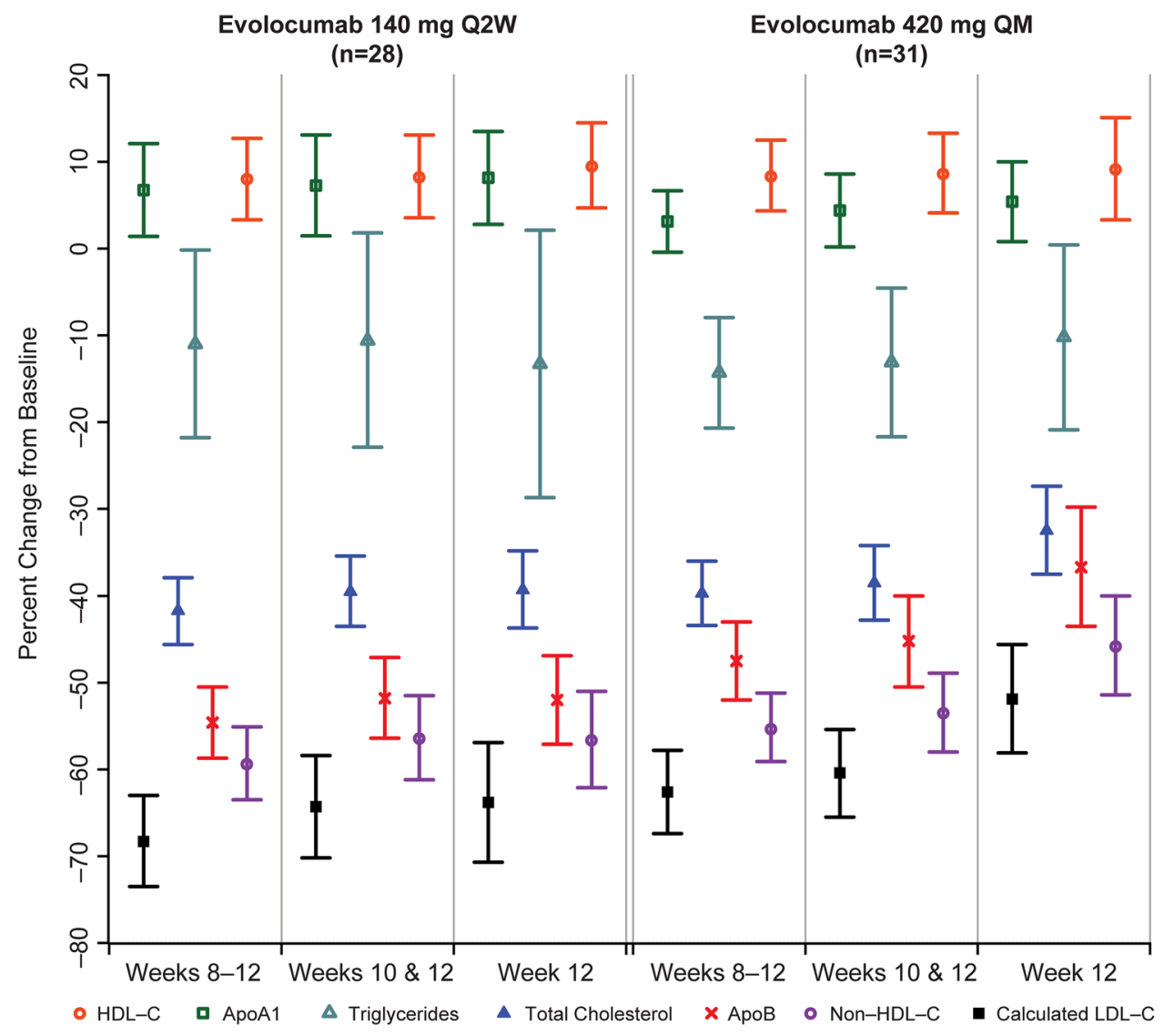

[29, 30]. After the 12-week study, these patients could enroll in a long-term extension study in which they received open-label evolocumab $420 \mathrm{mg}$ QM [44]. Mean \pm SD unbound evolocumab serum concentrations at open-label weeks 4,8 , and 12 (i.e., trough concentrations) were $9.31 \pm 6.92, \quad 12.7 \pm 11.1$, and $13.1 \pm 11.5 \mu \mathrm{g} / \mathrm{ml}$, respectively. Mean $\pm \mathrm{SD}$ unbound evolocumab serum concentrations at weeks 2,6 , and 10 (i.e., peak concentrations) were $38.6(n=1), 33.7(n=20.1)$, and 44.6 $(n=19.3) \mu \mathrm{g} / \mathrm{ml}$, respectively. Patients with HoFH who required lipid apheresis were not enrolled in the 12-week study but could enroll directly in the long-term extension study. These patients received a more frequent dose of evolocumab $420 \mathrm{mg}$ Q2W. Mean \pm SD unbound evolocumab serum concentrations pre-apheresis at open-label weeks $2,4,6,8,10$, and 12 (i.e., trough concentrations) ranged from $31.1 \pm 13.1$ to $77.0 \pm 27.2 \mu \mathrm{g} / \mathrm{ml}$ for these patients.

\subsection{Hepatic Impairment}

No dose adjustment is necessary in patients with mild/moderate hepatic impairment. Exposure to evolocumab after a single dose of $140 \mathrm{mg}$ was approximately $40-50 \%$ lower in patients with mild to moderate hepatic impairment (Child-Pugh A or B) than in patients with normal hepatic function [36]. Possible mechanisms for this observation are not known, and no statistically significant trends in evolocumab pharmacokinetics with advancing degree of hepatic impairment were noted. The pharmacodynamic profiles (LDL-C and PCSK9) and safety profile in patients with mild or moderate hepatic impairment were similar to those in patients with normal hepatic function.

\subsection{Renal Impairment}

No dose adjustment is necessary in patients with renal impairment. In a phase I single-dose study, exposure to evolocumab after a single dose of $140 \mathrm{mg}$ appeared to decrease in patients with severe renal impairment or endstage renal disease compared with patients with normal renal function [49]. Possible mechanisms for this observation are not known. The pharmacodynamic profiles (LDL-C and PCSK9) and safety profile in patients with severe renal impairment or end-stage renal disease were similar to those in patients with normal renal function. 
Table 1 Unbound evolocumab pharmacokinetic parameters for evolocumab monotherapy across patient populations in clinical studies [18, 20, 34]

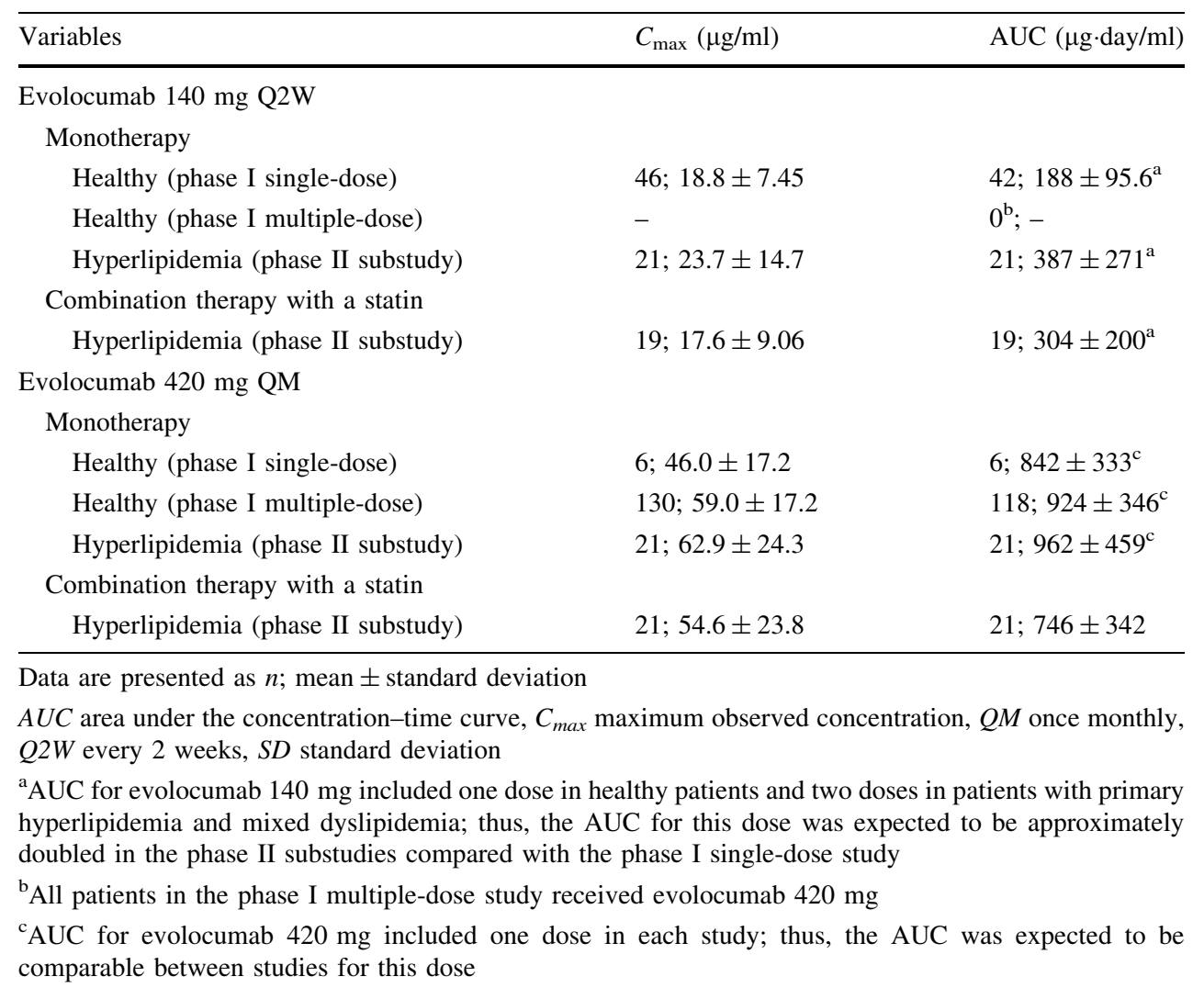

\subsection{Body weight}

No dose adjustment is necessary based on body weight. Evolocumab exposure tends to be lower in heavier patients than in lighter patients, but due to large inter-subject variability in exposure, there was extensive overlap in evolocumab exposures across the weight range of patients included in clinical studies (Fig. S1 in the Electronic Supplementary Material [ESM]). In cross-study comparisons across the initial phase II and III studies, no effect of body weight on LDL-C reduction was observed (Fig. S2 in the ESM).

\subsection{Race}

No dose adjustment is necessary based on race. In a phase I comparative study, healthy Japanese and healthy White subjects had similar pharmacokinetics and pharmacodynamics [35]. Across the initial phase II and III studies, pharmacokinetics and pharmacodynamics were similar across racial groups (Fig. S3 in the ESM).

\subsection{Sex}

No dose adjustment is necessary based on sex. Across the phase II and III studies, differences between female and male patients in median trough serum concentrations were approximately 48 and $18 \%$ higher for female patients who received subcutaneous evolocumab $140 \mathrm{mg}$ Q2W and subcutaneous evolocumab $420 \mathrm{mg}$ QM, respectively. Female patients had lower body weight than male patients and were therefore expected to have higher trough concentrations (Fig. S4 in the ESM). Pharmacodynamic effects (LDL-C reductions) were similar between men and women at these doses.

\subsection{Age}

No dose adjustment is necessary based on age. Across the initial phase II and III studies, no relationship was observed between age and pharmacokinetics or pharmacodynamics in patients aged 18-80 years (Fig. S5 in the ESM).

\subsection{Population Pharmacokinetics and Exposure- Response Relationships}

Data were pooled for 5468 patients in 11 clinical studies who received evolocumab to characterize population pharmacokinetics and predict LDL-C response in relation to exposure. Modeling confirmed evolocumab has nonlinear pharmacokinetics, and predictions suggested similar maximal LDL-C reduction of $66 \%$ at the mean of weeks 10 
and 12 for the $140 \mathrm{mg}$ Q2W and $420 \mathrm{mg}$ QM doses, as observed. The pharmacodynamic response at the extremes of intrinsic and extrinsic patient factors such as body weight and concomitant lipid-lowering therapy was predicted to be within $26 \%$ of the reference patient, which supported no dose adjustments for evolocumab therapy based on patient-specific factors. An exposure-response analysis was conducted to determine the relationships between evolocumab AUC over weeks 8-12 and mean of weeks 10 and 12 calculated LDL-C. The recommended doses of $140 \mathrm{mg}$ Q2W or $420 \mathrm{mg}$ QM led to a response near the plateau of the exposure-response relationship.

\section{Statin Drug-Drug Interactions}

Treatment with statins of differing doses and intensities was associated with lower exposure to unbound evolocumab, with an approximate $20 \%$ reduction in $C_{\max }$ and AUC compared with monotherapy (Table 1). This finding was consistent with evidence from other studies that statin treatment upregulates PCSK9 concentrations [50, 51], leading to increased binding of evolocumab and thus lower concentrations of unbound evolocumab. The pharmacodynamic effects of evolocumab on LDL-C are comparable between evolocumab monotherapy and adding evolocumab to a statin. Thus, the observed difference in pharmacokinetics among patients who receive evolocumab with a high-intensity statin regimen is not clinically meaningful and does not affect dosing recommendations.

\section{Summary}

Cardiovascular disease and dyslipidemia are highly prevalent causes of death and disability globally. Recent updates to clinical treatment guidelines recommended increased use of statin therapy in patients with dyslipidemia, but many patients need additional lipid lowering, are nonadherent to statin therapy, or are statin intolerant.

Evolocumab, a human monoclonal IgG2 that binds specifically to PCSK9, exhibits nonlinear kinetics. Peak serum concentrations are reached in 3-4 days. Approximately two- to threefold accumulation is observed in trough serum concentrations, which reach steady state by week 12 (i.e., after three $420 \mathrm{mg}$ QM doses or six $140 \mathrm{mg}$ Q2W doses). Evolocumab appears to have limited tissue distribution. Concentration-dependent binding between drug and target and subsequent elimination of the drugtarget complex augment the elimination of the drug over the endogenous elimination pathways such as catabolism by the reticuloendothelial system [40]. Elimination is predominantly through saturable binding to PCSK9 at lower concentrations and a non-saturable proteolytic pathway at higher concentrations. The effective half-life is 11-17 days.

Evolocumab is injected subcutaneously at a dose of either $140 \mathrm{mg}$ Q2W or $420 \mathrm{mg}$ QM. The pharmacodynamic effects of evolocumab on PCSK9 are rapid, with maximum suppression within $4 \mathrm{~h}$ of a single dose, whereas the nadir for LDL-C is more prolonged and occurs by 14 days for a single 140-mg dose and by 21 days for a single 420-mg dose. At steady state, the LDL-C nadir occurs approximately 1 week after a 140-mg Q2W dose and 2 weeks after a 420-mg QM dose. Neither PCSK9 nor LDL-C rebounds to a level above baseline after evolocumab washout. In numerous clinical studies, evolocumab reduced LDL-C by approximately 55-75\% compared with placebo at the mean of weeks 10 and 12 . The treatment effect was consistent across a variety of patient populations with dyslipidemia that received evolocumab for 12-52 weeks, either as monotherapy or added to another lipid-lowering therapy. A target-mediated drug disposition model further supported the therapeutic equivalence of $140 \mathrm{mg}$ Q2W or $420 \mathrm{mg}$ QM for LDL-C reduction.

No clinically meaningful differences in pharmacodynamic effects on LDL-C were observed in adult subjects regardless of mild/moderate hepatic impairment, renal impairment or renal failure, body weight, race, sex, or age. Concomitant use with a statin may decrease exposure to evolocumab, but no clinically meaningful differences were observed for the pharmacodynamic effects of evolocumab on LDL-C between patients who received evolocumab alone or in combination with a statin. No dose adjustment is necessary based on patient-specific factors or concomitant medication use. A recently completed placebo-controlled study of approximately 27,500 patients provided definitive evidence of the efficacy and safety of these doses of evolocumab to reduce the risk of cardiovascular events [48].

In conclusion, pharmacokinetic and pharmacodynamic data from 24 global clinical studies of evolocumab support the use of either $140 \mathrm{mg}$ Q2W or $420 \mathrm{mg}$ QM, based on prescriber and patient preference, to reduce LDL-C in patients with primary hyperlipidemia (familial or nonfamilial) and mixed dyslipidemia without the need for dose adjustment or titration.

Acknowledgements Medical writing assistance was provided by Jonathan Latham (PharmaScribe, LLC) and Meera Kodukulla (Amgen Inc.).

\section{Compliance with Ethical Standards}

Funding This work was supported by Amgen Inc. 
Conflict of interest Drs. Somaratne and Wasserman are employees of and stockholders in Amgen, Inc. Drs. Somaratne and J.P. Gibbs are identified as inventors on at least one pending patent application owned by Amgen, Inc. relating to evolocumab. Dr. Wasserman appears on a number of pending patents owned by Amgen Inc. relating to evolocumab and PCSK9 inhibition. Drs. Kasichayanula, Grover, Emery, M. Gibbs, and J.P. Gibbs were employees of and stockholders in Amgen at the time this work was completed. Drs. Kasichayanula, M. Gibbs, and J.P. Gibbs are currently employed by AbbVie, Inc. Dr. Grover is currently employed by BioMarin Pharmaceutical Inc. Dr. Emery is currently employed by Seattle Genetics.

Open Access This article is distributed under the terms of the Creative Commons Attribution-NonCommercial 4.0 International License (http://creativecommons.org/licenses/by-nc/4.0/), which permits any noncommercial use, distribution, and reproduction in any medium, provided you give appropriate credit to the original author(s) and the source, provide a link to the Creative Commons license, and indicate if changes were made.

\section{References}

1. World Health Organization. World health statistics 2012. Geneva: WHO Press; 2012.

2. Mozaffarian D, Benjamin EJ, Go AS, et al. Heart disease and stroke statistics-2015 update: a report from the American Heart Association. Circulation. 2015;131:e29-322.

3. Collaboration Cholesterol Treatment Trialists, Baigent C, Blackwell L, et al. Efficacy and safety of more intensive lowering of LDL cholesterol: a meta-analysis of data from 170,000 participants in 26 randomised trials. Lancet. 2010;376:1670-81.

4. Silverman MG, Ference BA, Im K, et al. Association between lowering LDL-C and cardiovascular risk reduction among different therapeutic interventions: a systematic review and metaanalysis. JAMA. 2016;316:1289-97.

5. Grundy SM, Cleeman JI, Merz CN, et al. Implications of recent clinical trials for the National Cholesterol Education Program Adult Treatment Panel III guidelines. Circulation. 2004;110:227-39.

6. Waters DD, Brotons $\mathrm{C}$, Chiang $\mathrm{CW}$, et al. Lipid treatment assessment project 2: a multinational survey to evaluate the proportion of patients achieving low-density lipoprotein cholesterol goals. Circulation. 2009;120:28-34.

7. Lemstra M, Blackburn D, Crawley A, et al. Proportion and risk indicators of nonadherence to statin therapy: a meta-analysis. Can J Cardiol. 2012;28:574-80.

8. Mancini GB, Baker S, Bergeron J, et al. Diagnosis, prevention, and management of statin adverse effects and intolerance: Canadian Consensus Working Group Update (2016). Can J Cardiol. 2016;32:S35-65.

9. Cannon CP, Blazing MA, Giugliano RP, et al. Ezetimibe added to statin therapy after acute coronary syndromes. N Engl J Med. 2015;372:2387-97.

10. Horton JD, Cohen JC, Hobbs HH. Molecular biology of PCSK9: its role in LDL metabolism. Trends Biochem Sci. 2007;32:71-7.

11. Brown MS, Goldstein JL. Biomedicine. Lowering LDL-not only how low, but how long? Science. 2006;311:1721-3.

12. Qian YW, Schmidt RJ, Zhang Y, et al. Secreted PCSK9 downregulates low density lipoprotein receptor through receptor-mediated endocytosis. J Lipid Res. 2007;48:1488-98.

13. Horton JD, Cohen JC, Hobbs HH. PCSK9: a convertase that coordinates LDL catabolism. J Lipid Res. 2009;50(Suppl):S172-7.
14. Abifadel M, Varret M, Rabes JP, et al. Mutations in PCSK9 cause autosomal dominant hypercholesterolemia. Nat Genet. 2003;34:154-6.

15. Cohen J, Pertsemlidis A, Kotowski IK, et al. Low LDL cholesterol in individuals of African descent resulting from frequent nonsense mutations in PCSK9. Nat Genet. 2005;37:161-5.

16. Lopez D. PCSK9: an enigmatic protease. Biochim Biophys Acta. 2008;1781:184-91.

17. Abifadel M, Rabes JP, Devillers M, et al. Mutations and polymorphisms in the proprotein convertase subtilisin kexin 9 (PCSK9) gene in cholesterol metabolism and disease. Hum Mutat. 2009;30:520-9.

18. Koren MJ, Scott R, Kim JB, et al. Efficacy, safety, and tolerability of a monoclonal antibody to proprotein convertase subtilisin/kexin type 9 as monotherapy in patients with hypercholesterolaemia (MENDEL): a randomised, double-blind, placebo-controlled, phase 2 study. Lancet. 2012;380:1995-2006.

19. Koren MJ, Lundqvist P, Bolognese M, et al. Anti-PCSK9 monotherapy for hypercholesterolemia: the MENDEL-2 randomized, controlled phase III clinical trial of evolocumab. J Am Coll Cardiol. 2014;63:2531-40.

20. Giugliano RP, Desai NR, Kohli P, et al. Efficacy, safety, and tolerability of a monoclonal antibody to proprotein convertase subtilisin/kexin type 9 in combination with a statin in patients with hypercholesterolaemia (LAPLACE-TIMI 57): a randomised, placebo-controlled, dose-ranging, phase 2 study. Lancet. 2012;380:2007-17.

21. Robinson JG, Nedergaard BS, Rogers WJ, et al. Effect of evolocumab or ezetimibe added to moderate- or high-intensity statin therapy on LDL-C lowering in patients with hypercholesterolemia: the LAPLACE-2 randomized clinical trial. JAMA. 2014;311:1870-82.

22. Raal F, Scott R, Somaratne R, et al. Low-density lipoprotein cholesterol-lowering effects of AMG 145, a monoclonal antibody to proprotein convertase subtilisin/kexin type 9 serine protease in patients with heterozygous familial hypercholesterolemia: the Reduction of LDL-C with PCSK9 Inhibition in Heterozygous Familial Hypercholesterolemia Disorder (RUTHERFORD) randomized trial. Circulation. 2012;126:2408-17.

23. Raal FJ, Stein EA, Dufour R, et al. PCSK9 inhibition with evolocumab (AMG 145) in heterozygous familial hypercholesterolaemia (RUTHERFORD-2): a randomised, double-blind, placebo-controlled trial. Lancet. 2015;385:331-40.

24. Sullivan D, Olsson AG, Scott R, et al. Effect of a monoclonal antibody to PCSK9 on low-density lipoprotein cholesterol levels in statin-intolerant patients: the GAUSS randomized trial. JAMA. 2012;308:2497-506.

25. Stroes E, Colquhoun D, Sullivan D, et al. Anti-PCSK9 antibody effectively lowers cholesterol in patients with statin intolerance: the GAUSS-2 randomized, placebo-controlled phase 3 clinical trial of evolocumab. J Am Coll Cardiol. 2014;63:2541-8.

26. Nissen SE, Stroes E, Dent-Acosta RE, et al. Efficacy and tolerability of evolocumab vs ezetimibe in patients with muscle-related statin intolerance: the GAUSS-3 randomized clinical trial. JAMA. 2016;315:1580-90.

27. Blom DJ, Hala T, Bolognese M, et al. A 52-week placebo-controlled trial of evolocumab in hyperlipidemia. N Engl J Med. 2014;370:1809-19.

28. Sabatine MS, Giugliano RP, Wiviott SD, et al. Efficacy and safety of evolocumab in reducing lipids and cardiovascular events. N Engl J Med. 2015;372:1500-9.

29. Stein EA, Honarpour N, Wasserman SM, et al. Effect of the proprotein convertase subtilisin/kexin 9 monoclonal antibody, AMG 145, in homozygous familial hypercholesterolemia. Circulation. 2013;128:2113-20. 
30. Raal FJ, Honarpour N, Blom DJ, et al. Inhibition of PCSK9 with evolocumab in homozygous familial hypercholesterolaemia (TESLA Part B): a randomised, double-blind, placebo-controlled trial. Lancet. 2015;385:341-50.

31. Stein EA, Giugliano RP, Koren MJ, et al. Efficacy and safety of evolocumab (AMG 145), a fully human monoclonal antibody to PCSK9, in hyperlipidaemic patients on various background lipid therapies: pooled analysis of 1359 patients in four phase 2 trials. Eur Heart J. 2014;35:2249-59.

32. Nicholls SJ, Puri R, Anderson T, et al. Effect of evolocumab on progression of coronary disease in statin-treated patients: the GLAGOV randomized clinical trial. JAMA. 2016;316:2373-84.

33. Chan JC, Piper DE, Cao Q, et al. A proprotein convertase subtilisin/kexin type 9 neutralizing antibody reduces serum cholesterol in mice and nonhuman primates. Proc Natl Acad Sci USA. 2009;106:9820-5.

34. Dias CS, Shaywitz AJ, Wasserman SM, et al. Effects of AMG 145 on low-density lipoprotein cholesterol levels: results from 2 randomized, double-blind, placebo-controlled, ascending-dose phase 1 studies in healthy volunteers and hypercholesterolemic subjects on statins. J Am Coll Cardiol. 2012;60:1888-98.

35. Teramoto T, Shaywitz A, Hamilton L, et al. A phase 1, randomized, double-blind, placebo-controlled study of AMG 145 comparing the pharmacokinetics, pharmacodynamics, and safety in healthy Japanese and Caucasian subjects. In: Proceedings of 45th annual scientific meeting of the Japan Atherosclerosis Society, Tokyo; 2013.

36. Gibbs JP, Slatter JG, Egbuna O, et al. Evaluation of evolocumab (AMG 145), a fully human anti-PCSK9 IgG2 monoclonal antibody, in subjects with hepatic impairment. J Clin Pharmacol. 2017;57:513-23.

37. Lee WP, Datta BN, Ong BB, et al. Defining the role of lipoprotein apheresis in the management of familial hypercholesterolemia. Am J Cardiovasc Drugs. 2011;11:363-70.

38. Hirayama A, Honarpour N, Yoshida M, et al. Effects of evolocumab (AMG 145), a monoclonal antibody to PCSK9, in hypercholesterolemic, statin-treated Japanese patients at high cardiovascular risk-primary results from the phase 2 YUKAWA study. Circ J. 2014;78:1073-82.

39. REPATHA ${ }^{\circledR}$ (evolocumab) Prescribing Information. Thousand Oaks: Amgen, Inc.; 2016.

40. Gibbs JP, Doshi S, Kuchimanchi M, et al. Impact of target-mediated elimination on the dose and regimen of evolocumab, a human monoclonal antibody against proprotein convertase subtilisin/kexin type 9 (PCSK9). J Clin Pharmacol. 2017;57:616-26.

41. Colbert A, Umble-Romero A, Prokop S, et al. Characterization of a quantitative method to measure free proprotein convertase subtilisin/kexin type 9 in human serum. MAbs. 2014;6:1103-13.

42. Koren MJ, Doshi S, Castro R, et al. Comparisons of peak LDL-C reduction and duration of effect with lower or higher dosing regimens of the PCSK9 inhibitor evolocumab [abstract]. Circulation. 2015;132:A12729.

43. Rosenson RS, Jacobson TA, Preiss D, et al. Efficacy and safety of the PCSK9 inhibitor evolocumab in patients with mixed hyperlipidemia. Cardiovasc Drugs Ther. 2016;30:305-13.

44. Raal FJ, Hovingh GK, Blom D, et al. Long-term treatment with evolocumab added to conventional drug therapy, with or without apheresis, in patients with homozygous familial hypercholesterolaemia: an interim subset analysis of the open-label TAUSSIG study. Lancet Diabetes Endocrinol. 2017;5:280-90.

45. Raal FJ, Giugliano RP, Sabatine MS, et al. PCSK9 inhibitionmediated reduction in $L p(a)$ with evolocumab: an analysis of 10 clinical trials and the LDL receptor's role. J Lipid Res. 2016;57:1086-96.

46. Watts GF, Chan DC, Dent R, et al. Factorial effects of evolocumab and atorvastatin on lipoprotein metabolism. Circulation. 2017;135:338-51.

47. Desai NR, Giugliano RP, Wasserman SM, et al. Association between circulating baseline proprotein convertase subtilisin kexin type 9 levels and efficacy of evolocumab. JAMA Cardiol. 2017;2:556-60.

48. Sabatine MS, Giugliano RP, Keech AC, et al. Evolocumab and clinical outcomes in patients with cardiovascular disease. N Engl J Med. 2017;376:1713-22.

49. Lee E, Gibbs J, Wasserman SM, et al. Pharmacokinetics and pharmacodynamics of evolocumab in patients with renal impairment [abstract]. Eur Heart J. 2016;37(Suppl):343.

50. Dubuc G, Chamberland A, Wassef $\mathrm{H}$, et al. Statins upregulate PCSK9, the gene encoding the proprotein convertase neural apoptosis-regulated convertase-1 implicated in familial hypercholesterolemia. Arterioscler Thromb Vasc Biol. 2004;24:1454-9.

51. Mayne J, Dewpura T, Raymond A, et al. Plasma PCSK9 levels are significantly modified by statins and fibrates in humans. Lipids Health Dis. 2008;7:22.

52. Brown MS, Goldstein JL. Receptor-mediated endocytosis: insights from the lipoprotein receptor system. Proc Natl Acad Sci USA. 1979;76:3330-7.

53. Steinberg D, Witztum JL. Inhibition of PCSK9: a powerful weapon for achieving ideal LDL cholesterol levels. Proc Natl Acad Sci USA. 2009;106:9546-7.

54. Goldstein JL, Brown MS. The LDL receptor. Arterioscler Thromb Vasc Biol. 2009;29:431-8.

55. Zhang DW, Lagace TA, Garuti R, et al. Binding of proprotein convertase subtilisin/kexin type 9 to epidermal growth factor-like repeat A of low density lipoprotein receptor decreases receptor recycling and increases degradation. $J$ Biol Chem. 2007;282:18602-12. 